\section{Hipoxemia espuria por hiperleucocitosis. Reporte de un caso y revisión de la literatura}

\author{
PABLO FLORENZANO V..$^{1-2}$, LUZ MARÍA LETELIER S. ${ }^{1,2}$, \\ JOSEFINA DURÁN S. C. ${ }^{3}$, CRISTÓBAL SANHUEZA C. ${ }^{4}$, LUIS TORO C. ${ }^{5}$
}

\section{Spurious hypoxemia due to hyperleukocytosis. Report of one case}

Arterial gasometry is considered the gold standard for establishing a diagnosis of respiratory failure of any etiology. However, there are some circumstances in which it loses specificity, making necessary to consider other tests such as pulse oximetry to adequately determine hypoxemia. We report a 67 years old patient with sudden hypoacusia, right hemiparesis and polypnea. His laboratory exams on admission, showed extreme hypoxemia in several readings, without correlation to the patient's clinical condition nor the pulse oximetry, and a leukocytosis of 800.000 cells $x \mathrm{ml}$, with many immature cells. Chronic myeloid leukemia was diagnosed and treatment with hydroxyurea was initiated, achieving normalization in the arterial gases in accordance with the fall of the white cell count. Interpretation of laboratory findings according to the general clinical context of the patient allowed to suspect a spurious hypoxemia, saving the patient from unnecessary and risky interventions.

(Rev Med Chile 2012; 140: 503-506).

Key words: Anoxia; Leukemia, myeloid; Leukocytosis.

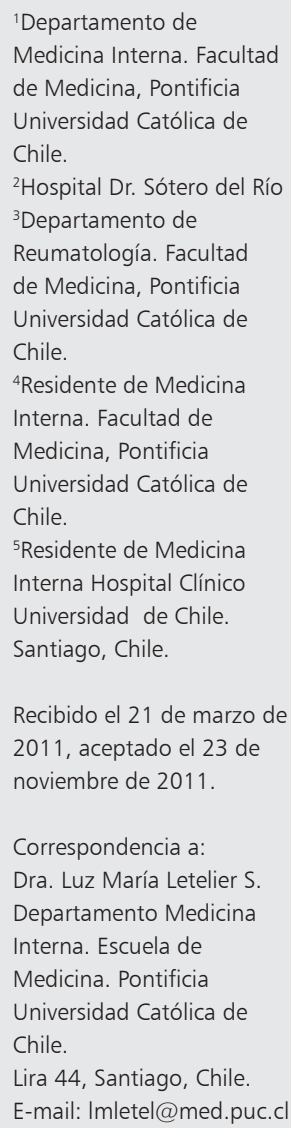

L a hiperleucocitosis es la elevación patológica del recuento de leucocitos en la sangre, gecúbico ${ }^{1}$.Frecuentemente secundaria a procesos mieloproliferativos o leucemias agudas, pueden asociarse a un Síndrome de hiperviscosidad y/o a un Síndrome de lisis tumoral constituyendo urgencias oncológicas. Existe un fenómeno menos conocido que se puede presentar en los pacientes con hiperleucocitosis. Ésta es la alteración espuria de los gases arteriales, específicamente la disminución de la presión arterial de oxígeno $\left(\mathrm{P}_{\mathrm{a}} \mathrm{O}_{2}\right)$ en ausencia de síntomas o signos de hipoxia y sin correlación con la Oximetría de pulso del paciente. Este fenómeno se denomina hipoxemia espuria. Esta alteración, de no ser considerada, puede llevar a una interpretación errónea de la condición del paciente, derivando en tratamientos agresivos y potencialmente dañinos.
Presentamos un caso clínico de hipoxemia espuria.

\section{Caso clínico}

Paciente hombre de 67 años, con antecedentes de etilismo crónico. Cinco semanas antes del ingreso presentó súbitamente hipoacusia bilateral. El día del ingreso presentó paresia braquio-crural derecha de instalación brusca, asociada a compromiso cualitativo de conciencia, por lo cual fue llevado a Urgencia del Hospital Dr. Sótero del Río.

$\mathrm{Al}$ examen físico se constató vigil, desorientado, con Glasgow $14\left(\mathrm{~V}_{4} \mathrm{M}_{6} \mathrm{O}_{4}\right)$; hemodinámicamente estable, afebril, polipneico (30/min); bien hidratado y perfundido, sin cianosis, muy enflaquecido. Examen pulmonar con crepitaciones bibasales simétricas; corazón con ruidos rítmicos en dos tiempos, sin soplos; abdomen blando, depresible, 
hígado palpable a $2 \mathrm{~cm}$ bajo reborde costal y bazo palpable $10 \mathrm{~cm}$ bajo reborde costal de consistencia normal. El examen neurológico mostró déficit motor braquio-crural derecho $\left(\mathrm{M}_{3}\right.$ miembro superior e inferior), con reflejo plantar extensor ipsilateral, sin signos meníngeos.

\section{Exámenes de laboratorio}

Hcto: $32,4 \%$; Leucocitos: $800.000 \mathrm{x} \mathrm{mm}^{3}$ (12\% baciliformes, $8 \%$ juveniles, $15 \%$ mielocitos); Plaquetas: $135.000 \times \mathrm{xm}^{3}$; Creatininemia: $1,63 \mathrm{mg} /$ dL; BUN: 13 mg/dL; VHS: 3 mm/h; LDH: 2.155 U/L (Rango normal Laboratorio 135-214 U/L); Ácido úrico: $12 \mathrm{mg} / \mathrm{dL}$; Calcio: 8,1 mEq/L; INR: 1,49 TTPK: 32 seg.

Radiografía de Tórax: infiltrado intersticial bilateral de predominio basal; sin cardiomegalia.

\section{Gases arteriales de ingreso}

$$
\begin{aligned}
& \mathrm{P}_{\mathrm{a}} \mathrm{O}_{2}: 3 \mathrm{mmHg} \\
& \mathrm{F}_{\mathrm{i}} \mathrm{O}_{2}: 0,21 \\
& \mathrm{~S}_{\mathrm{a}}: 1 \% \\
& \mathrm{P}_{\mathrm{a}} \mathrm{CO}_{2}: 53 \mathrm{mmHg} \\
& \text { pH: } 7,20 \\
& \mathrm{HCO}_{3}: 20 \mathrm{mEq} / \mathrm{L}
\end{aligned}
$$

Debido a la marcada alteración gasométrica, discordante con la clínica y la oximetría de pulso ( $93 \%$ con $\mathrm{F}_{i} \mathrm{O}_{2}$ ambiental) se repitió toma de gases arteriales, trasladando la muestra en hielo en un tiempo cercano a los 3 minutos, obteniéndose:

$$
\begin{aligned}
& \text { Gases arteriales de control } \\
& \mathrm{P}_{\mathrm{a}} \mathrm{O}_{2}: 65 \mathrm{mmHg} \\
& \mathrm{S}_{\mathrm{a}}: 93 \% \\
& \mathrm{P}_{\mathrm{a}} \mathrm{CO}_{2}: 33 \mathrm{mmHg} \\
& \mathrm{pH}: 7,20 \\
& \mathrm{HCO}_{3}: 19,4 \mathrm{mEq} / \mathrm{L}
\end{aligned}
$$

Se interpretaron los resultados de los gases arteriales como espurios, secundarios a hiperleucocitosis, por lo cual no se consideraron para definir el manejo del paciente.

Se hospitalizó con los diagnósticos de probable leucemia mieloide crónica (LMC), hipoacusia y hemiparesia secundaria a síndrome de hiperviscosidad, iniciando tratamiento con hidroxiurea e hidratación endovenosa con suero fisiológico, presentando mejoría cualitativa de conciencia.

El recuento leucocitario descendió progresivamente (Tabla 1), con mejoría de los niveles de $\mathrm{P}_{\mathrm{a}} \mathrm{O}_{2}$ y $\mathrm{S}_{2} \mathrm{O}_{2}$ proporcionales al descenso de leucocitos (Figura 1), alcanzando niveles normales al $10^{\circ}$ día. Es importante destacar que sus valores de glicemia y kalemia se mantuvieron normales durante toda la hospitalización. Se demostró translocación cromosómica 9:22, confirmándose el diagnóstico de LMC.

El paciente evolucionó consciente, orientado, en Glasgow 15, persistiendo con hipoacusia severa bilateral y paresia braquio-crural derecha, sin cambios respecto al ingreso. Se mantiene con tratamiento para LMC con hidroxiurea, indicándose el alta hospitalaria con plan de control ambulatorio.

\section{Discusión}

Si bien no se conoce su incidencia real ${ }^{2}$ las alteraciones espurias de los gases arteriales son eventos observados con cierta frecuencia por los clínicos. La gran mayoría se origina por problemas en la toma de muestra: uso de frascos inadecuados para la recolección, presencia de burbujas de aire en la muestra, demora en el traslado al laboratorio o hemólisis por agitación del frasco, entre las más frecuentes ${ }^{3,4}$. Sin embargo, existen también alteraciones espurias de la gasometría debido a condiciones propias del paciente, como es el caso de la hiperleucocitosis. Si bien las hipoxemias espurias son eventos infrecuentes ${ }^{5}$, tienen gran importancia clínica ya que su desconocimiento puede determinar conductas activas dirigidas a tratar la "insuficiencia respiratoria" con aporte de altas fracciones inspiradas de $\mathrm{O}_{2} \mathrm{o}$ apoyo ventilatorio invasivo, que ponen en riesgo el bienestar y la vida del paciente.

Existe escasa información sobre hipoxemia espuria en relación a leucocitosis en la literatura. Una búsqueda en PubMed, usando la estrategia de búsqueda: "Anoxemia"[Mesh] AND "Leukocytosis"[Mesh] arrojó 19 artículos relevantes, siendo 11 de ellos reporte de casos sobre pacientes que presentaron esta alteración.

En estos reportes se encontró que todos los pacientes que presentan esta alteración tienen una elevación patológica del recuento leucocitario, causada más frecuentemente por una leucemia mieloide crónica. Se describen además casos aislados en que las alteraciones de los gases arteriales se asociaron a trombocitosis 6 .

La etiopatogenia postulada por Chillar et $\mathrm{al}^{5}$ sería el consumo de oxígeno por parte de las células 
Tabla 1. Resumen de exámenes realizados al paciente

\begin{tabular}{|lcccccc|}
\hline & Día 1 & Día 3 & Día 8 & Día 11 & Día 13 & Día 15 \\
\hline Leucocitos $\left(\mathrm{cel} / \mathrm{mm}^{3}\right)$ & 800.000 & 810.000 & 442.000 & 240.000 & 101.000 & 71.500 \\
\hline $\mathrm{PaO}_{2}(\mathrm{mmHg})$ & 3 & 3 & 10 & 35 & 57 & 70 \\
$\mathrm{SaO}_{2}(\%)$ & 1 & 1 & 5 & 20 & 89 & 95 \\
$\mathrm{PaCO}_{2}(\mathrm{mmHg})$ & 53 & 52 & 33 & 25 & 25 & 32 \\
$\mathrm{pH}$ & 7,2 & 7,2 & 7,41 & 7,47 & 7,29 & 7,34 \\
\hline
\end{tabular}

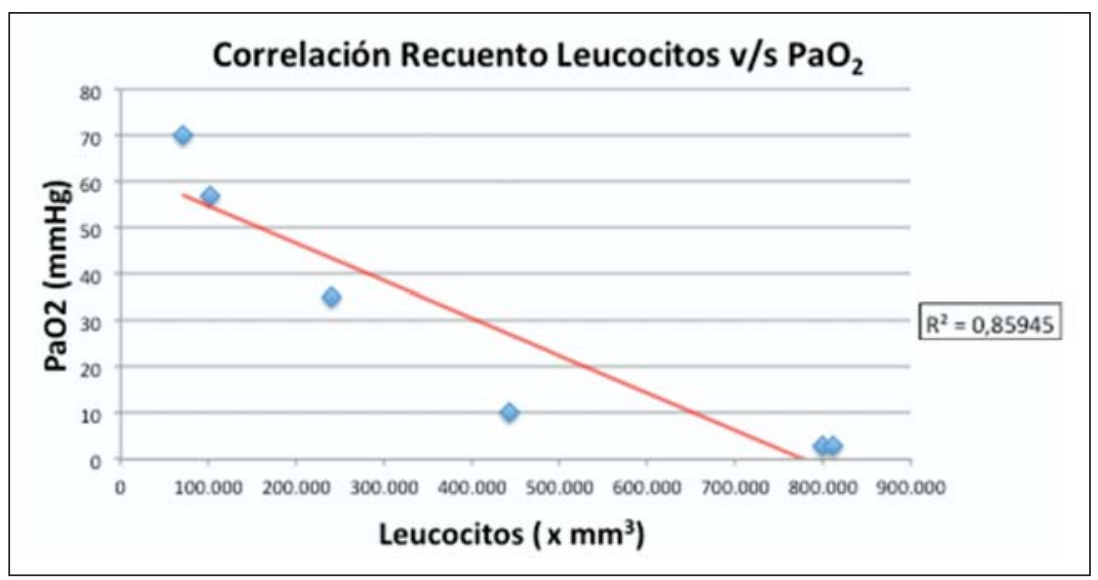

Figura 1. Correlación entre recuento de leucocitos sanguíneos y $\mathrm{P}_{2} \mathrm{O}_{2}$ del paciente. Línea Roja: Regresión lineal de la correlación $\left(R^{2}: 0,85\right)$. hematológicas, el cual estaría aumentado por el elevado metabolismo de las células neoplásicas. El consumo aumentado de $\mathrm{O}_{2}$ disminuiría el contenido de oxígeno en la muestra en el tiempo transcurrido desde la toma de la muestra hasta el análisis en el laboratorio, mostrando una hipoxemia espuria en la sangre arterial. Sin embargo, "in vivo" esta disminución de $\mathrm{O}_{2}$ plasmática no es tan severa como para producir hipoxia, en ausencia de otras patologías (neumonía, sepsis, alteraciones ventilación/perfusión pulmonar), debido probablemente a que el tiempo de traslado de la muestra al laboratorio es mayor que el período entre la oxigenación de la sangre y la entrega de $\mathrm{O}_{2}$ al tejido; esto explicaría la ausencia de signos y síntomas de hipoxia en los pacientes descritos ${ }^{7}$, presentando oximetría de pulso en rangos normales ${ }^{8,9}$, la cual mide la $\mathrm{S} \mathrm{O}_{2}$ in situ. Este mecanismo explicaría además el ascenso de la $\mathrm{PO}_{\mathrm{a}}$ en una relación lineal ${ }^{1}$ con la reducción leucocitaria secundaria a quimioterapia. Todo lo anteriormente expuesto es concordante con lo descrito en nuestro caso.
También se describen en estos pacientes otras alteraciones en exámenes de laboratorio ${ }^{1,8}$, como hipoglicemia, acidosis metabólica o respiratoria e hiperkalemia, en ausencia de clínica compatible u otras causas que las originen. Éstas serían explicadas por el aumento del consumo y/o excreción de sustancias por las células neoplásicas.

Es importante destacar que, debido a que los pacientes con hiperleucocitosis presentan una patología severa en evolución asociada con una deficiencia del sistema inmune (secundaria a la misma enfermedad o al tratamiento), la posibilidad real de que presenten una hipoxemia, una acidosis o una hiperkalemia verdadera, secundaria a los procesos patológicos propios de estos pacientes, tales como infección o síndrome de lisis tumoral, es muy alta. Por esto el enfrentamiento inicial es complejo y debe ser siempre acorde a la clínica del paciente y a la posibilidad real de presentar estas alteraciones.

Cuando sea posible se debe tomar la muestra y procesarla al lado de la cama del paciente. Si esto 
no es posible deberán tomarse las precauciones necesarias para procesar la muestra lo más rápidamente posible.

En el estudio de Lele et $\mathrm{al}^{1}$, se propone un algoritmo para el enfrentamiento de la hipoxemia en el paciente con hiperleucocitosis. Siguiendo este algoritmo, frente a un paciente con hiperleucocitosis que presente hipoxemia $\left(\mathrm{PO}_{2}<60 \mathrm{mmHg}\right.$ ), acidosis, hipoglicemia o hiperkalemia, se debe evaluar dirigidamente al paciente en búsqueda de signos o síntomas concordantes, y complementar con otros exámenes si es necesario:

- Oximetría de pulso si se sospecha hipoxemia.

- Hemoglucotest en el caso de hipoglicemia.

- ECG si se sospecha hiperkalemia.

Si no se encuentran alteraciones concordantes con los exámenes de laboratorio se debe sospechar una alteración espuria y no se deben considerar estos resultados en la toma de decisiones clínicas.

Finalmente, una vez iniciada la quimioterapia y confirmada la reducción leucocitaria, se deben evaluar en forma seriada los exámenes para confirmar la normalización de éstos en relación a la disminución del recuento leucocitario; de no ser así, se debe descartar otras etiologías que originen estas alteraciones.

\section{Conclusión}

Si bien la alteración espuria de los gases arteriales es una manifestación poco frecuente de patologías que cursan con hiperleucocitosis, es fundamental reconocerla en el enfrentamiento de pacientes con este tipo de patologías. Es vital realizar una adecuada correlación entre la clínica y la gasometría arterial, apoyándose siempre en las oxímetría de pulso. De identificarse una alteración espuria de la gasometría no debe considerarse en el manejo del paciente.

\section{Referencias}

1. Lele AV, Mirski MA, Stevens RD. Spurious hypoxemia. Crit Care Med 2005; 33: 1854-6.

2. Khoo SM, Lee KH, Notley M. Spurious hypoxaemia in a patient with leukaemia and extreme leucocytosis. Singapore Med J 2003; 44:208-10.

3. Gorski TF, Ajemian M, Hussain E, Talhouk A, Ruskin G, Hanna A, et al. Correlation of pseudohypoxemia and leukocytosis in chronic lymphocytic leukemia. South Med J 1999; 92:817-9.

4. Mizock BA, Franklin C, LindesmithI P, Shah PC. Confirmation of spurious hypoxemia using continuous blood gas analysis in a patient with chronic myelogenous leukemia. Leuk Res 1995; 19: 1001-4.

5. Chillar RK, Belman MJ, Farbstein M. Explanation for apparent hypoxemia with extreme leukocytosis: leukocytic oxygen consumption. Blood 1980; 55: 922-4.

6. Chillar RK, Belman MJ, Farbstein M. Pseudohypoxemia due to leukemia and thrombocytosis. N Engl J Med 1980; 302: 584-5.

7. Gartrell K, Rosenstrauch W. Hypoxaemia in patients with hyperleukocytosis: true or spurious, and clinical implications. Leuk Res 1993; 17: 915-9.

8. Sacchetti A, Grynn J, Pope A, Vasso S. Leukocyte larceny: spurious hypoxemia confirmed with pulse oximetry. J Emerg Med 1990; 8: 567-9.

9. Loke J, Duffy TP. Normal arterial oxygen saturation with the ear oximeter in patients with leukemia and leukocytosis. Cancer 1984; 53: 1767-9. 